ISSN 1997-5902

\title{
Impact des activités anthropiques sur la dynamique de la végétation de la réserve partielle de faune de Pama et de ses périphéries (sud-est du Burkina Faso) dans un contexte de variabilité climatique
}

\author{
Soungalo Soulama1, 2*, Abel Kadeba², Blandine M.I. Nacoulma², Salifou Traoré2, Yvonne Bachmann³, \\ Adjima Thiombiano ${ }^{2}$ \\ 1. Département Productions Forestières, INERA, CNRST 03 BP 476 Ouagadougou 03, Burkina Faso. \\ E.mail:soulsoung@yahoo.fr \\ 2. Laboratoire de Biologie et Écologie Végétales, Université de Ouagadougou, 03 BP 7021 Ouagadougou 03. \\ 3. J. W. Goethe University, Institute of Ecology, Evolution and Diversity Max-von-Laue-Str. 13, 60438 Frankfurt am \\ Main Germany. \\ *Auteur correspondant : Soungalo Soulama ; Email : soulsoung@yahoo.fr
}

Original submitted in on 21st November 2014. Published online at www.m.elewa.org on 31st March 2015 http://dx.doi.org/10.4314/jab.v87i1.6

\section{RÉSUMÉ}

Objectif: Ce travail réalisé dans la réserve partielle de faune de Pama et ses périphéries a pour objectif d'évaluer les changements opérés dans la végétation sous l'influence du climat et de l'Homme.

Méthodologie et résultats : Des images Landsat de 2001 et 2013 ont été utilisées pour faire la classification de la végétation. Une ordination des sites en fonction des paramètres de végétation collectées, a permis de faire le lien entre les sites dégradées et leur mode d'utilisation. Les variations du climat sont analysées à partir de l'indice standardisé des précipitations, les séquences sèches et le nombre de jours pluvieux. Les résultats indiquent une réduction des formations végétales denses, et une augmentation de la végétation savanicole, sols nus et mosaïques champs-jachères. La carte typologique des changements d'occupation du sol, a montré une amélioration de $66,8 \%$ de couverture végétale localisée surtout dans les aires protégées tandis que la portion de la zone d'étude dégradée est de $28,8 \%$. Les paramètres pluviométriques montrent que la décennie 2001-2010 est relativement plus aride que celle précédente. Quant à l'impact des modes d'utilisation des terres, les aires pâturés et les jachères sont les plus dégradées comparativement à la Réserve partielle de faune de Pama et aux forêts communautaires.

Conclusion et application : La dégradation des écosystèmes de cette zone résulte d'une action combinée des facteurs climatiques et anthropiques. Les phytocénoses situées dans les aires protégées sont les moins dégradées, ce qui réaffirme l'importance de ces aires de conservation dans un contexte de pression anthropique et de péjoration climatique.

Mots clés : dégradation des terres; images Landsat; indice de précipitations; types d'utilisation des terres. 


\section{ABSTRACT}

Objective: This work done in Pama partial wildlife reserve and its surroundings, aims to assess the changes in vegetation under climate and humans disturbances.

Methodology and results: Landsat images from 2001 and 2013 were used for vegetation classification. Sites ordination according to vegetation parameters, allowed making link between degraded sites and how to use them. Climate variations are analyzed from the standardized precipitation index, dry sequences and number of rainy days. The results indicate a reduction of dense vegetation and increasing of savannahs, bare soil and mosaics of fields or fallows. Change detection map, showed an improvement of $66.8 \%$ mainly localized vegetation in protected areas while the portion of degraded sites in study area is $28.8 \%$. Rainfall parameters show that the decade 2001-2010 is relatively drier than the previous. As for the impact of land use patterns, grazed lands and fallows are the most degraded compared to Pama partial Wildlife Reserve and community forests.

Conclusion and Application: Ecosystem degradation of this area is the result of a combined action of climatic and anthropogenic factors. Ecosystems located in protected areas are the least degraded, which reaffirms the importance of conservation areas in the context of study area.

Keywords: land degradation; Landsat images; Precipitation Index; Land use types.

\section{INTRODUCTION}

La dégradation des terres est l'un des plus graves problèmes environnementaux contemporains. Elle est définie comme la perte de productivité biologique des terres (Reynolds \& Stafford Smith, 2002; FAO, 2008). Les savanes d'Afrique connaissent des processus rapides de transformation des paysages ruraux et une dégradation des ressources naturelles (Ariori \& Ozer, 2005; Larwamou et al., 2005; Mama et al., 2013). La question de savoir le primat entre l'action de l'homme et celle du climat dans la dégradation des terres au sahel, préoccupe les scientifiques (Nicholson, 2005; Olsson et al, 2005; Reij et al., 2009). L'indice standardisé des précipitations (SPI), est souvent utilisé pour apprécier l'aridité climatique d'une zone. Le calcul de cet indice permet de déterminer le degré d'humidité ou de sécheresse du milieu (Bergaoui \& Alouini, 2001). II est bien adapté au suivi des variations de la dynamique de la végétation en relation avec l'évolution de la pluviométrie (Diallo et al., 2011). Jadis épargnées par le phénomène de dégradation des terres en raison de la faible pression anthropique, les zones sud soudaniennes du Burkina Faso sont désormais soumises à une crise environnementale. La migration interne de populations des zones dégradées vers celles favorables aux activités agro-pastorales contribuent à accélérer la dégradation de ces dernières (Youl et al, 2008; Ouédraogo et al., 2009; Ouédraogo et al., 2010). C'est le cas dans la zone du sud-est du Burkina Faso qui abrite d'importantes aires de conservation, où diverses études font cas de la dégradation des ressources naturelles (Mbaygone et al, 2008; Ouédraogo, 2009; Nacoulma et al., 2011). L'ampleur de la pression foncière dans cette région peut s'observer à travers l'installation des populations dans des enclaves à l'intérieur de la réserve partielle de faune de Pama et l'occurrence des conflits d'usage (Kaboré, 2010; Kpoda, 2010). Hahn-Hadjali (1998) souligne que presque tous les groupements végétaux de la zone sont soumis à l'influence anthropozoïque. Les changements d'occupation des terres sont souvent connus mais c'est leur ampleur qui diffère selon le type de pression et les conditions écologiques et environnementales. A cet effet, la télédétection permet d'effectuer l'étude de la dynamique de la dégradation et des causes sous-jacentes à ce phénomène (Schmid, 2003; Geymen \& Baz, 2008; Paré et al., 2010). La détection du changement des unités d'occupation du sol est possible grâce à la technique de postclassification et l'établissement des matrices de 
transition (Shalaby \& Tateishi, 2007; Maârouhi et al., 2011). Compte tenu de l'importance du complexe d'aires protégées de l'Est du Burkina Faso dans la conservation de la diversité biologique, de nombreuses études ont été effectuées (Hahn-Hadjali, 1998; Mbaygone et al., 2008; Ouédraogo et al., 2008; Sawadogo et al, 2014). En dépit de ces efforts, les dynamiques de déforestation sont rarement mises en évidence, d'où l'absence de cartes d'évolution spatiotemporelle de la dégradation au niveau local. La présente étude vise à combler cette insuffisance. Elle a pour objectif général d'analyser la dynamique de changements d'occupation des sols en vue de déterminer les causes de la dégradation des phytocénoses, dans la réserve partielle de

\section{MATÉRIEL ET MÉTHODE}

Zone d'étude : L'aire d'investigation se situe à cheval sur les provinces du Gourma et de la Kompienga, à l'Est du Burkina Faso (Figure 1). La zone d'étude est située en grande partie dans le secteur soudanien septentrional, avec son extrême sud dans le district sud faune de Pama et les agrosystèmes avoisinants. Les objectifs spécifiques sont de: (i) cartographier les unités d'occupation des sols dans la réserve partielle de faune de Pama et les agrosystèmes adjacents; (ii) déterminer l'impact des facteurs pluviométriques dans la dégradation des terres de la zone d'étude; (iii) évaluer l'impact des types d'utilisation des terres dans la dynamique des agrosystèmes de la zone d'étude. Notre hypothèse est que les changements opérés au cours de ces dix dernières années sur le couvert végétal dans la réserve partiel de faune de Pama et ses agrosystèmes périphériques, sont fonction d'un gradient d'anthropisation dans un contexte de péjoration climatique.

soudanien (Fontès \& Guinko, 1995). Son régime pluviométrique est monomodal, caractérisé par des précipitations moyennes annuelles qui oscillent entre 700 et $1000 \mathrm{~mm}$ et une température variant entre $25^{\circ} \mathrm{C}$ et $39^{\circ} \mathrm{C}$.

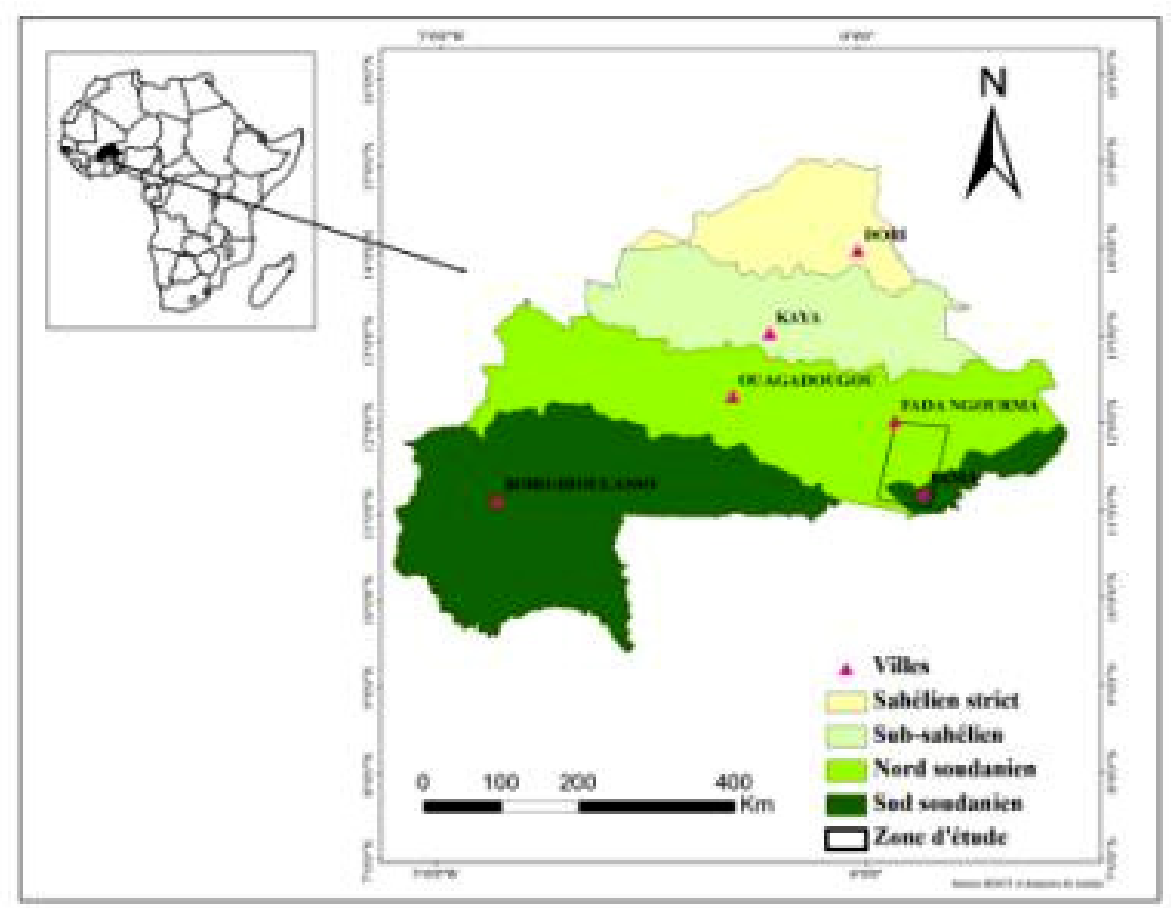

Figure 1: localisation de la zone d'étude 
Les altitudes varient entre 250 et $300 \mathrm{~m}$ avec les plus faibles valeurs $(160 \mathrm{~m})$ aux bordures de la rivière Pendjari. Les principaux sols sont de types ferrugineux tropicaux lessivés peu profonds à profonds. On note également la présence de vertisols peu répandus, de sols hydromorphes le long des berges de cours d'eau. Les savanes arbustives et arborées dominent le paysage environnant. Ces savanes sont parsemées d'îlots de formations forestières claires et sillonnées par des savanes boisées, forêts claires et forêts galeries, surtout dans les abords des cours d'eau (Fontes \& Guinko, 1995; Hahn-Hadjali, 1998; Mbaygone, et al., 2008). Du point de vue du statut, la réserve partielle de faune de Pama est dans la catégorie VI (UICN, 2009), c'est-à-dire une aire protégée ouverte aux populations pour un ensemble défini d'usages (paille, médicaments). Les variantes anthropisées des formations végétales sont dominées par des mosaïques de champs-jachères; des aires de pâturage et des forêts communautaires. Ces dernières, appelées " zones villageoises d'intérêt cynégétiques » (ZOVIC), sont règlementées par une gestion participative entre plusieurs villages limitrophes. Le statut des ZOVIC permet leur usage pour la chasse et le pâturage. Mais les activités d'agriculture, ou de coupe de bois ainsi que la pratique des feux de brousse sont formellement interdites (Kaboré, 2010). La dynamique de la population dans la zone d'étude est importante. En effet, les taux de croissance de la population du Gourma et de la Kompienga entre 1985 et 2010, sont respectivement de $37,72 \%$ et $45,26 \%$ (INSD, 2008).

\section{Collecte des données}

Données images: Les images satellitaires utilisées sont de type Landsat ETM+, scènes 193/052 du 20 octobre 2001 et du 19 octobre 2013. La résolution spatiale de ces images est de 30 mètres, de projection UTM zone $31 \mathrm{~N}$ avec ellipsoïde de référence WGS84. Les images acquises à cette période apparaissent les plus appropriées car les cultures champêtres ne perturbent pas la signature spectrale de la végétation naturelle et les feux de végétation dans la forêt de Pama ne sont pas encore passés.

Données climatiques : Les données pluviométriques concernent les hauteurs pluviométriques des stations météorologiques de Fada N'Gourma (district sudsoudanien) et de Pama (district nord soudanien), de 1953 à 2013.

Données de végétation: Un échantillonnage stratifié et aléatoire a été adopté pour la collecte des données de végétation. Le critère de classification est le mode d'utilisation des terres. Les quatre principaux types d'utilisation des terres sont la réserve partielle de faune de Pama (FCPN), les forêts communautaires (ZOVIC), les mosaïques champs-jachères (FALL) et les aires de pâturage (GZLD). Ces dernières concernent aussi bien les espaces de pâturage des villages, les pistes de transhumance du bétail, que les points d'eau d'abreuvement. Au total 377 placettes principales de $50 \mathrm{~m} \times 50 \mathrm{~m}$ ont été visitées. Sur chacune, le type de végétation a été noté selon la typologie de Yangambi (Aubreville,1957), en même temps que les informations stationnelles et les mesures dendrométriques et floristiques. Dans chaque placette principale, des sous placeaux de $10 \mathrm{~m} \times 10 \mathrm{~m}$ pour les herbacées et $5 \mathrm{~m} \times$ $5 \mathrm{~m}$ pour les individus de la régénération $(\mathrm{dbh}<5 \mathrm{~cm}$ ). Les relevés de végétation effectués dans chaque placette, comprennent: la richesse spécifique moyenne des ligneux adultes, de la régénération et des herbacées; le taux de couverture herbacée et de buissons; la hauteur moyenne des arbustes et des herbes; le diamètre moyenne des arbres; la densité (nombre d'individus / ha) des ligneux et des individus de la régénération ainsi que la surface terrière. La surface terrière des peuplements (pour les individus dont le $\mathrm{dbh} \geq 5 \mathrm{~cm}$ ) est calculée suivant la formule:

$$
S T C=\frac{10000 \pi}{4 z} \sum_{i=1}^{n} d t^{2}
$$

Avec STC: surface terrière en $\mathrm{m}^{2} /$ ha; di: diamètre de l'arbre i en m; s: $1000 \mathrm{~m}^{2}$.

\section{Analyse des données}

Traitement des images: Des opérations de corrections géométriques et radiométriques ont permis de régler le calage entre les deux scènes et d'améliorer leur lisibilité (Tabopda et al., 2010; Diallo et al., 2011). Les bandes 54 et 3 ont été utilisées pour la composition colorée car elles caractérisent mieux la couverture végétale. La classification supervisée à l'aide de l'algorithme du maximum de vraisemblance, a été utilisée grâce à la connaissance du terrain acquise. Par ailleurs, pour éliminer les pixels isolés et homogénéiser la classification thématique, un filtre de 3 x 3 pixels a été appliqué aux images classifiées. Par classe d'occupation, 30 échantillons ont été utilisés pour la vérification. Les résultats du test de vérification donnent les matrices de confusion qui confrontent les résultats cartographiques et les données collectées sur le terrain. La précision globale est mesurée par le coefficient Kappa (Pointus, 2000; Koné et al., 2007; 
Maârouhi et al., 2011). Le traitement des images et leur analyse ont été faits à l'aide des logiciels ENVI 4.7 et ArcGIS 9.3.

Identification et typologie des zones de changement: Le traitement et la vectorisation des images sont effectués suivis de l'analyse spatiale, sont effectués à l'aide des logiciels ENVI 4.7 et ArcGIS 9.3. Le croisement des deux cartes d'occupation du sol (2001 et 2013) a permis d'établir la carte de dynamique des unités d'occupation du sol durant ces 12 dernières années. Étant donné la place de la thématique forestière dans cette étude, pour la détection du changement une codification des classes basée sur la nomenclature de la FAO (2001) est utilisée pour apprécier le changement de la végétation (Tableau 1). Une soustraction est effectuée entre les valeurs digitales des deux images de telle sorte que la détection du changement a consisté à identifier le changement de codes pour les vecteurs homologues $(\Delta i)$. Une matrice de transition générée à partir des analyses spatiales, décrit les changements d'état des classes thématiques pendant la période considérée (2001-2013).

Tableau 1: typologie des changements des unités d'occupation du sol

\begin{tabular}{|c|c|c|}
\hline $\begin{array}{l}\text { Variation } \\
\text { du code } \Delta(i)\end{array}$ & $\begin{array}{l}\text { Typologie } \\
\text { du changement }\end{array}$ & Description \\
\hline-3 & Déforestation & $\begin{array}{l}\text { Destruction de formations ligneuses denses ou conversion en d'autres types } \\
\text { d'utilisation des terres }\end{array}$ \\
\hline-2 & Déboisement & $\begin{array}{l}\text { Conversion des forêts en un autre type d'utilisation des terres qui réduit le couvert } \\
\text { forestier à moins de } 10 \%\end{array}$ \\
\hline-1 & Dégradation & $\begin{array}{l}\text { Légère affection des formations ligneuses qui se traduit par une réduction du couvert } \\
\text { forestier ou du matériel sur pied }\end{array}$ \\
\hline 0 & Zone stable & Sans changement \\
\hline 1 & Amélioration & $\begin{array}{l}\text { Légère affection des formations ligneuses qui se traduit par un léger accroissement } \\
\text { du couvert forestier ou du matériel sur pied. }\end{array}$ \\
\hline 2 & Boisement & $\begin{array}{l}\text { Le rétablissement des formations forestières après une situation où le couvert } \\
\text { forestier était inférieur à } 10 \% \text {. }\end{array}$ \\
\hline$>3$ & Reboisement & $\begin{array}{l}\text { Le rétablissement au cours d'une décennie, d'au moins } 10 \% \text { du couvert forestier } \\
\text { détruit après une perturbation. }\end{array}$ \\
\hline
\end{tabular}

Évaluation de l'impact climatique: La formule de l'indice standardisé des précipitations (SPI) est la suivante:

$S P I=\frac{x_{i}-x_{m \mathrm{~m}}}{s_{i}}$. Avec,

$X i$ valeur de la pluviométrie de l'année i;

Xm: valeur moyenne interannuelle de la pluviométrie sur la période étudiée;

Si: l'écart- type des pluies annuelles observées pour la série concernée.

Lorsque SPI $>2$, on parle d'humidité extrême ; pour $1<$ $\mathrm{SPI}<2$, on a une humidité forte; pour $0<\mathrm{SPI}<1$, on a une humidité modérée; pour $-1<\mathrm{SPI}<0$, on a une sécheresse modérée ; si $-2<\mathrm{SPI}<-1$, on a une sécheresse forte ; si $\mathrm{SPI}<-2$, la sécheresse est qualifiée d'extrême. Pour déterminer les localités qui présentent une chronique pluviométrique similaire,, une
ACP a permis de superposer les variables (paramètres pluviométriques) et les individus (localités /décennie) conformément à la démarche utilisée par Sinsin (1993). Les paramètres pluviométriques utilisées sont la pluviométrie, le nombre de jours pluvieux, l'indice standardisé des précipitations, les séquences sèches i.e. le nombre de jours consécutifs sans pluies.

Évaluation de l'impact des types d'utilisation des terres sur la dégradation: Une ordination des sites (ACP) en fonction des paramètres de végétation collectés, a servi à décrire leurs caractéristiques écologiques et structurales, à déceler ceux qui sont dégradés et à faire le lien entre l'état de dégradation des sites et le mode d'utilisation des terres. Les analyses statistiques sont réalisées à l'aide des logiciels "R"et SPSS 18.0. 
Soulama et al. J. Appl. Biosci. Impact des activités anthropiques sur la dynamique de la végétation de la réserve partielle de faune de Pama et de ses périphéries (sud-est du Burkina Faso) dans un contexte de variabilité climatique

\section{RÉSULTATS}

Vérification des classifications: Sept classes communes d'occupation du sol ont finalement été cartographiées (Figure 2). Elles sont représentées par les forêts galerie, les forêts claires, les savanes boisées, les savanes arbustives ou arborées, les mosaïques de jachères et champs, les sols nus et affleurement rocheux et les plans d'eau. La discrimination entre les différentes classes thématiques

est statistiquement significative avec des précisions statistiques globales de $95,3 \%$ à $91,4 \%$ et des coefficients Kappa de 0,94 et 0,89 respectivement pour les images de 2001 et 2013. Les matrices de confusion montrent globalement qu'il n'existe pas d'énormes erreurs de confusion ni d'omission entre les classes (Tableaux 2 et 3 ).

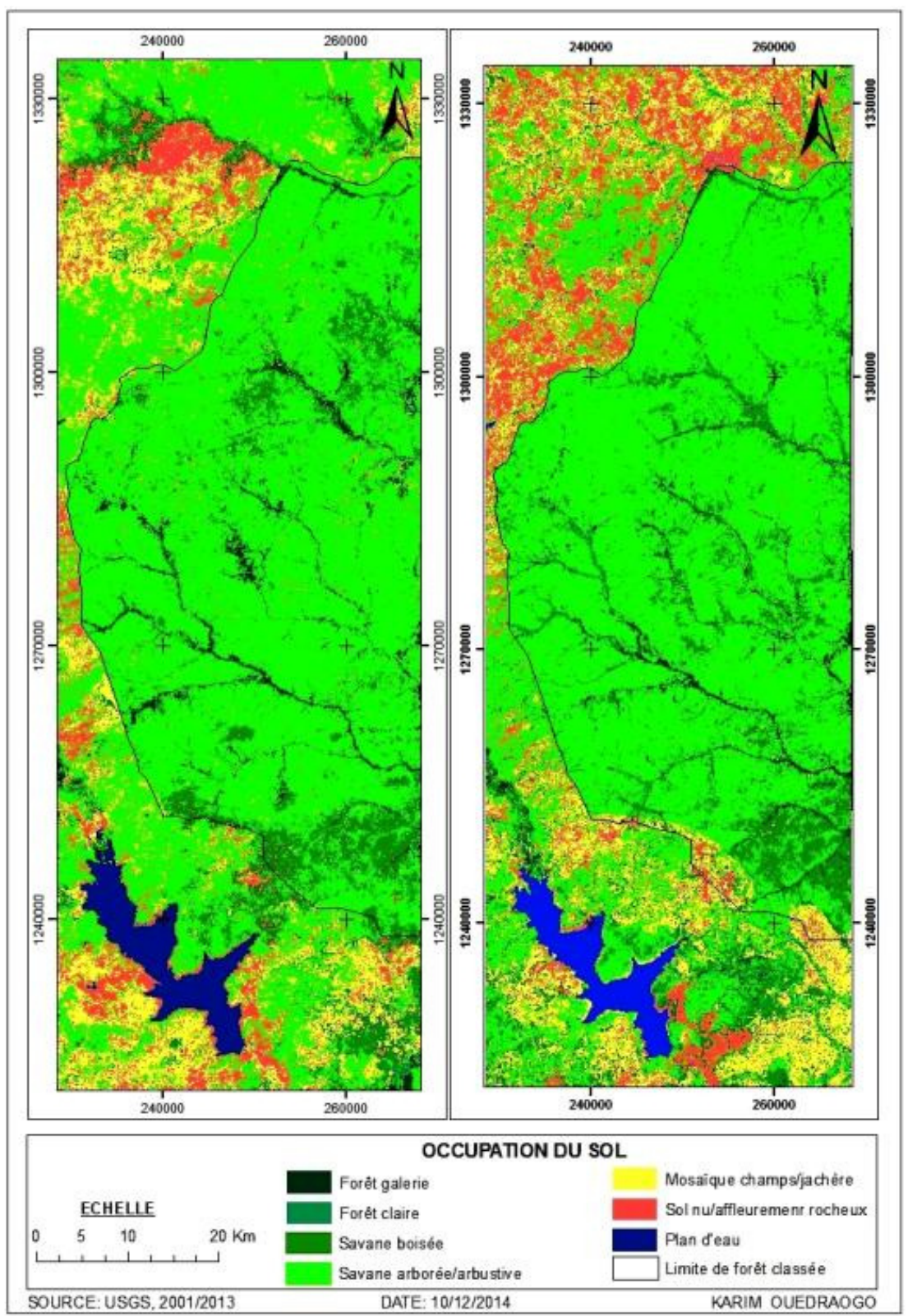

Figure 2: Occupation du sol en 2001 et en 2013 dans la réserve de Pama et sa périphérie 


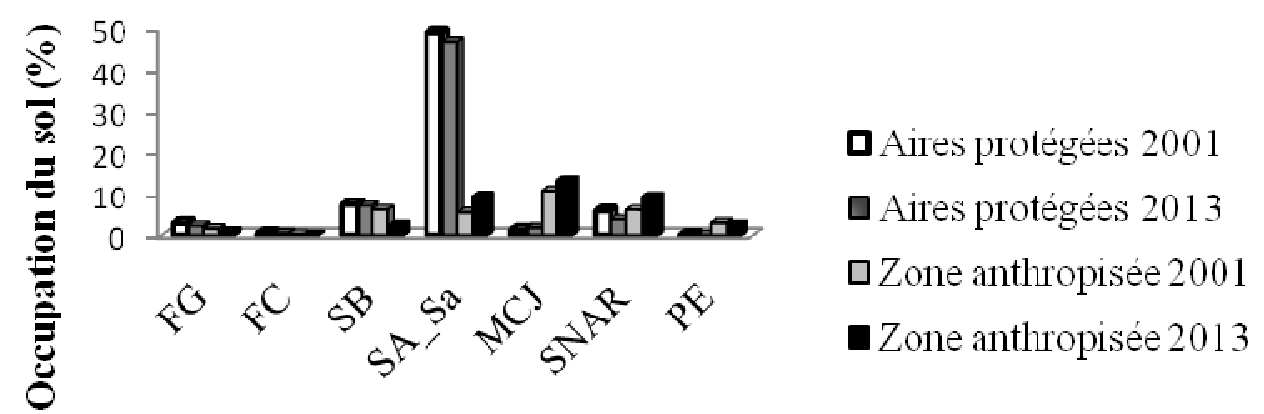

\section{Unités d'occupation du sol}

Figure 3: Dynamique de l'occupation du sol entre 2001 et 2013 dans la zone d'étude

Tableau 2: Matrice de confusion de la classification de l'image de 2001

\begin{tabular}{lllllllll}
\hline & $\mathbf{1}$ & $\mathbf{2}$ & $\mathbf{3}$ & $\mathbf{4}$ & $\mathbf{5}$ & $\mathbf{6}$ & $\mathbf{7}$ & EO \\
\hline $\mathbf{1}$ & $\mathbf{9 9 , 2}$ & 0 & 3,7 & 0 & 0 & 0 & 1,55 & 0,84 \\
$\mathbf{2}$ & 0 & $\mathbf{1 0 0}$ & 0,2 & 0 & 0 & 0 & 0 & 0 \\
$\mathbf{3}$ & 0,8 & 0 & $\mathbf{9 5 , 5}$ & 0 & 0 & 0 & 0 & 4.47 \\
$\mathbf{4}$ & 0 & 0 & 0 & $\mathbf{9 1 , 4}$ & 0 & 0 & 9,69 & 8,57 \\
$\mathbf{5}$ & 0 & 0 & 0,6 & 3,7 & 100 & 0 & 0 & 0 \\
$\mathbf{6}$ & 0 & 0 & 0 & 2 & 0 & 99,65 & 0 & 0,35 \\
7 & 0 & 0 & 0 & 0 & 2,9 & 0,35 & $\mathbf{8 8 , 7 6}$ & 11,24 \\
EC & 16,31 & 1,33 & 0,2 & 10,04 & 11,65 & 1,74 & 3,38 & \\
\hline
\end{tabular}

1: Forêt galerie; 2: Forêt claire; 3: Savane boisée ; 4: Savane arborée/arbustive ; 5 : Mosaïque champs/jachères; 6 : sol nu/affleurement rocheux; 7: Plan d'eau; EO: erreur d'omission; EC: erreur de commission

Tableau 3 : Matrice de confusion de la classification de l'image de 2013

\begin{tabular}{lllllllll}
\hline & $\mathbf{1}$ & $\mathbf{2}$ & $\mathbf{3}$ & $\mathbf{4}$ & $\mathbf{5}$ & $\mathbf{6}$ & $\mathbf{7}$ & EO \\
\hline $\mathbf{1}$ & $\mathbf{1 0 0}$ & 0 & 0 & 1,2 & 0 & 0 & 18,5 & 0 \\
$\mathbf{2}$ & 0 & 100 & 0,7 & 0 & 0 & 0 & 0 & 0 \\
$\mathbf{3}$ & 0 & 0 & 95 & 0 & 17,1 & 0 & 0 & 5 \\
$\mathbf{4}$ & 0 & 0 & 1,7 & 92,9 & 0 & 0 & 1,8 & 7,1 \\
$\mathbf{5}$ & 0 & 0 & 2,7 & 5,8 & $\mathbf{8 2 , 9}$ & 0 & 0 & 17,1 \\
$\mathbf{6}$ & 0 & 0 & 0 & 0 & 0 & 96,5 & 0 & 3,5 \\
$\mathbf{7}$ & 0 & 0 & 0 & 0,2 & 0 & 3,5 & 79,7 & 20,3 \\
EC & 34,4 & 1,2 & 17,8 & 1,2 & 21 & 0 & 5,3 & \\
\hline
\end{tabular}

1: Forêt galerie; 2: Forêt claire; 3: Savane boisée ; 4: Savane arborée/arbustive ; 5: Mosaïque champs/jachères; 6 : sol nu/affleurement rocheux; 7: Plan d'eau; EO: erreur d'omission; EC: erreur de commission

État d'occupation des sols en 2001 et 2013 : L'analyse des transferts d'occupation entre 2001 et 2013 montre essentiellement deux phénomènes: la réduction et l'ouverture du paysage. La réduction concerne les formations végétales denses (forêts galerie, forêts claires et savanes boisées) qui sont converties en savanes arbustives ou arborées (Figure 3). Cela est confirmé par la matrice de transition (Tableau 4) qui indique une conversion des forêts galeries, forêts claires et savanes boisées en savanes 
arbustive et arborée dans des proportions respectives de $2,8 \% ; 0,3 \%$ et $3,4 \%$. Les savanes arbustive et arborée constituent la classe d'occupation la plus dominante dans la zone d'étude (Figure 3). L'ouverture du paysage est remarquable dans la partie anthropisée où les sols nus et les mosaïques de champs-jachères se développent alors que ces mêmes classes thématiques ont tendance à se réduire dans la forêt
(Figure 2). En effet, dans toute la zone et en 12 ans, la conversion en mosaïques champs-jachères, concerne $4,1 \%$ de savane boisée et $4,7 \%$ de savane arborée et arbustive. Dans le même temps ces deux précédentes classes de végétations sont converties en sols nus et affleurement rocheux dans les taux respectifs de $1,7 \%$ et 3,3\% (Tableau 4).

Tableau 4: Matrice de transition en pourcentage d'occupation des sols dans la réserve de Pama et ses périphéries

\begin{tabular}{|c|c|c|c|c|c|c|c|c|c|}
\hline & & \multicolumn{8}{|c|}{2013} \\
\hline \multirow{9}{*}{2001} & Classes & FG & $\mathrm{FC}$ & SB & SAA & MCJ & SAR & PE & TOTAL \\
\hline & $\overline{F G}$ & 0.5 & 0.2 & 0.4 & 2.8 & 0.2 & 0.3 & 0.0 & 4.4 \\
\hline & FC & 0.1 & 0.1 & 0.1 & 0.3 & 0.1 & 0.0 & 0.0 & 0.6 \\
\hline & SB & 1.4 & 0.2 & 2.6 & 3.4 & 4.1 & 1.7 & 0.0 & 13.4 \\
\hline & SAA & 0.9 & 0.2 & 5.2 & 40.2 & 4.7 & 3.3 & 0.2 & 54.6 \\
\hline & MCJ & 0.1 & 0.0 & 0.4 & 4.6 & 4.4 & 2.4 & 0.1 & 12.1 \\
\hline & SAR & 0.2 & 0.0 & 0.7 & 4.1 & 1.6 & 5.2 & 0.1 & 12.0 \\
\hline & PE & 0.0 & 0.0 & 0.0 & 0.4 & 0.1 & 0.3 & 2.1 & 3.0 \\
\hline & TOTAL & 3.1 & 0.7 & 9.6 & 55.9 & 15.2 & 13.0 & 2.5 & 100.0 \\
\hline
\end{tabular}

1: Forêt galerie; 2: Forêt claire; 3: Savane boisée ; 4: Savane arborée/arbustive dense; 5: Savane arborée/arbustive peu dense; 6 : Mosaïque champs/jachères; 7 : sol nu/affleurement rocheux.

Typologie et répartition spatiale des changements intervenus : La typologie des changements intervenus (Figure 4) montre qu'au cours de cette décennie 0,2\% de la surface de la zone d'étude, autrefois constitués de formations ligneuses plus ou moins denses ont complètement disparu et ont été converties en terres arables (déforestation); $1,8 \%$ du secteur sont déboisés et $28,8 \%$ de cette superficie sont concernés par la dégradation des formations ligneuses. En somme, 30,8 $\%$ du paysage forestier est dégradé. A l'opposé de cette tendance, on observe que $2,4 \%$ de l'aire d'étude n'ont subi aucun changement, pendant que $66,8 \%$ de cette zone connaît une amélioration de la couverture ligneuse répartie comme suit:58,3 \% d'amélioration (recrût forestier); 7,9\% de boisement modéré et 0,7 \% de l'aire d'étude où la forêt s'est reconstituée (reboisement). D'une manière générale, les zones ayant connu une dégradation sont davantage localisées dans la partie anthropisée tandis que celles ayant connu une amélioration forestière sont situées dans la réserve de Pama et dans les ZOVIC (Figure 4). 


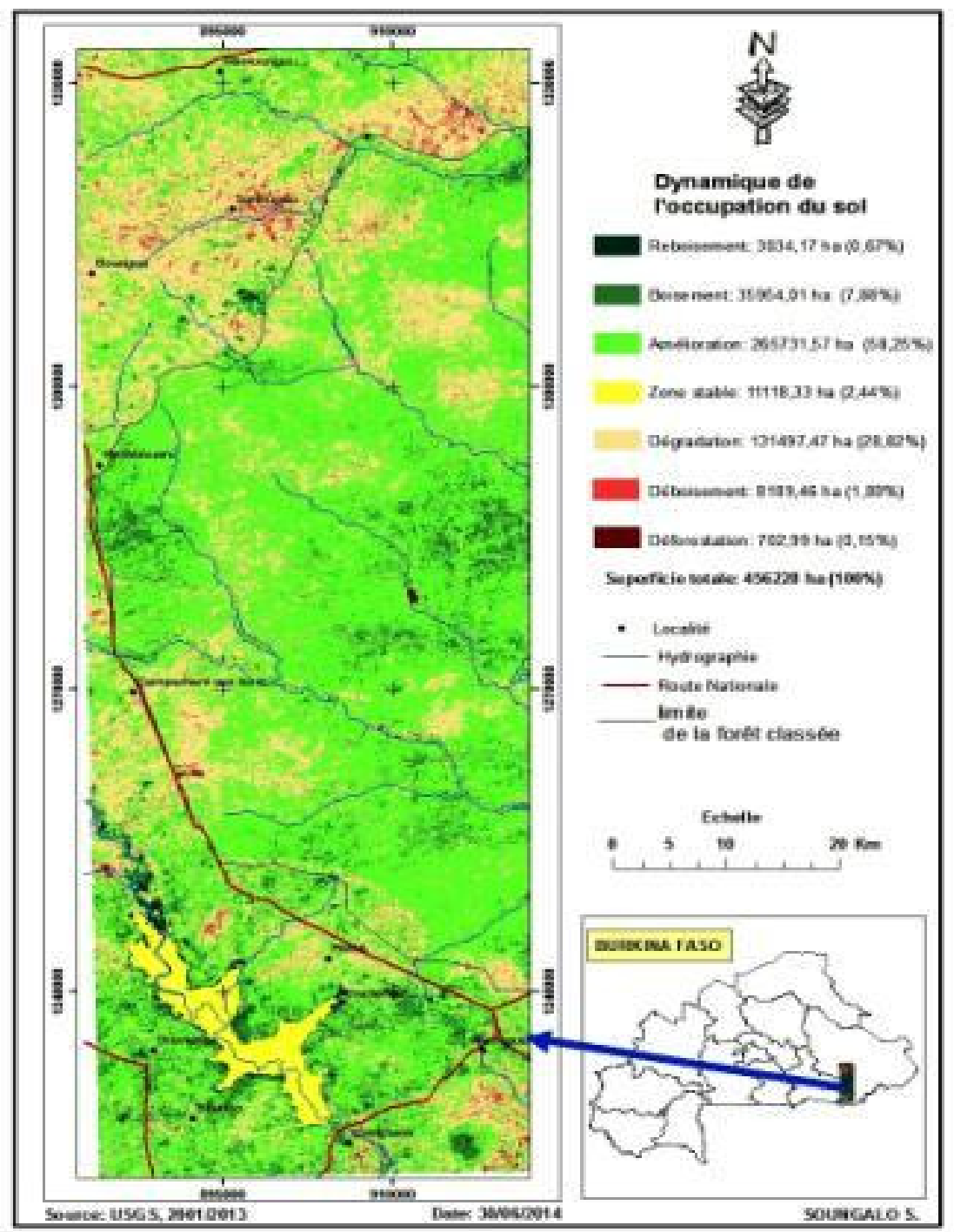

Figure 4: Dynamique de changement du couvert végétal dans la réserve de pama et ses agrosystèmes avoisinants

Évolution climatique : Les figures $5 a$ et $5 b$ montrent l'évolution des périodes sèches/humides respectivement dans les zones de Fada N'Gourma et de Pama. La tendance générale des indices pluviométriques est à la baisse avec une péjoration permanente des valeurs négatives à partir de la fin de la décennie 1960. En effet, les années 1950 enregistrent globalement une séquence humide qui marque l'abondance des précipitations, tandis qu'autour des années 1973, 1985 et 2000, on note des sécheresses relativement fortes. La période de 19812010 , est marquée par une alternance cyclique entre les années d'humidité et les années de sécheresse avec toutefois des épisodes secs de plus en plus longs. Les sécheresses enregistrées au cours de cette période dans les deux districts phytogéographiques, sont majoritairement modérées et quelquefois fortes, les extrêmes sont rares. 


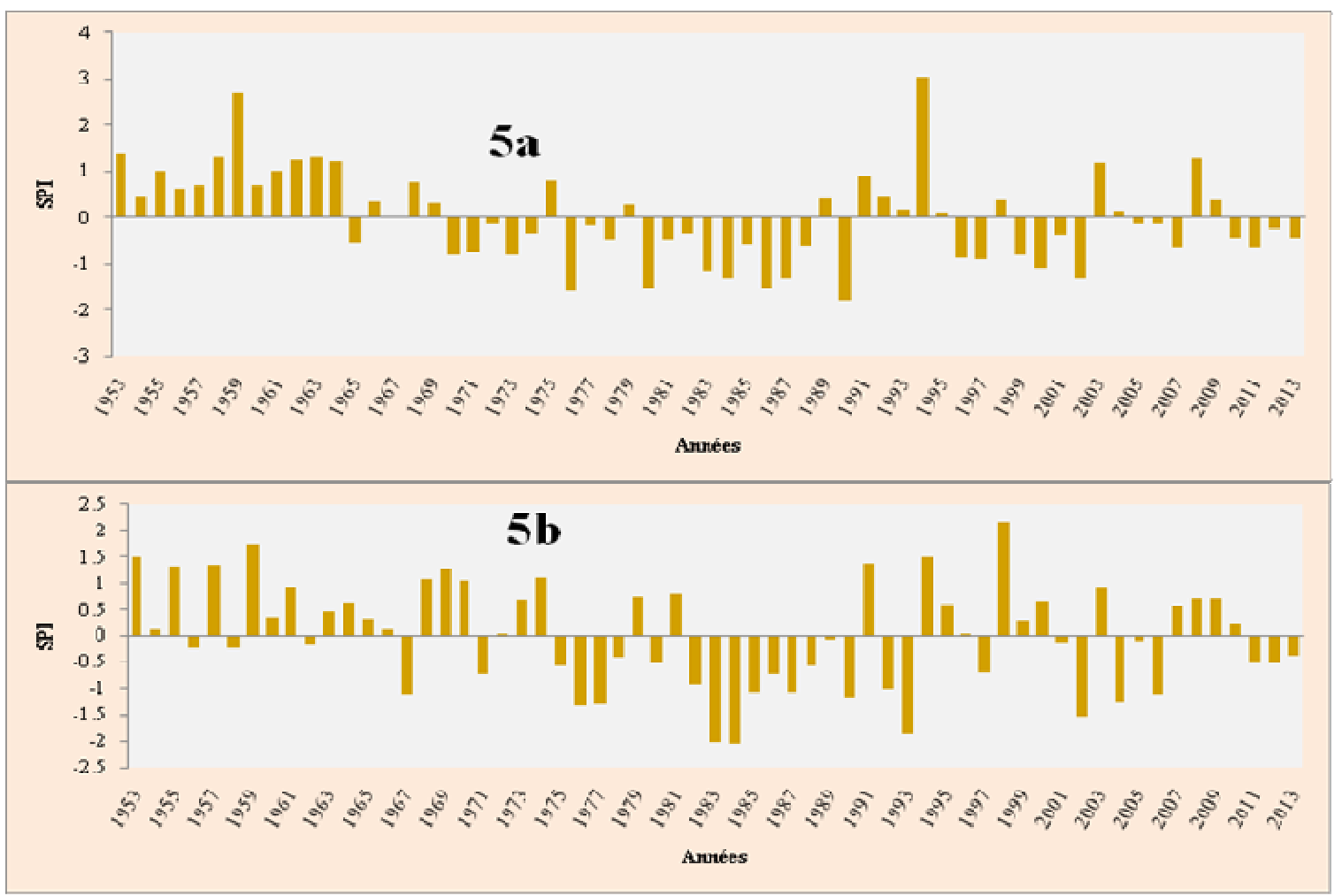

Figures $5 a$ et $5 b$ : Indices pluviométriques standardisés (SPI) dans la station de Fada N'Gourma (5a) et de Pama (5b).

Une analyse en composantes principales (ACP) indique que les deux premiers axes expliquent $99,6 \%$ de la variabilité entre les individus (localités selon les trois périodes climatiques de la période de référence 19812010). La superposition des projections de variables (paramètres pluviométriques) et des individus (localités/période) dans le plan formé par les 2 axes permet de définir l'axe 1 comme étant celui qui indique l'état de sécheresse et l'axe 2 comme étant celui de la répartition pluviométrique (Figures $6 \mathrm{a}$ et $6 \mathrm{~b}$ ). Ainsi, la période 1 (1981-1990) se distingue des deux autres périodes par son aridité avec des séquences sèches très élevées. Les grandes sécheresses des années 1985 ont été encore plus sévères à Pama. Quant à la période 2 (1991-2000) et la période 3 (2001-2010), elles sont relativement plus humides mais la décennie 2001-2010 serait modérément plus sèche que la précédente (Tableau 5). Cette sécheresse modérée est ressentie aussi bien au niveau des valeurs des séquences sèches, des hauteurs des pluies annuelles et du nombre de jours de pluie $(P<0)$. Par ailleurs, la zone de Pama au cours de la décennie 2001-2010, apparaît plus sèche que celle de Fada N' Gourma (Figures $5 a, 5 b$ et $6 b$ ). Cette sécheresse relative peut être corrélée avec la dynamique régressive de la végétation observée dans la Figure 2, aussi bien dans les aires protégées que dans les parties anthropisées. 
Soulama et al. J. Appl. Biosci. Impact des activités anthropiques sur la dynamique de la végétation de la réserve partielle de faune de Pama et de ses périphéries (sud-est du Burkina Faso) dans un contexte de variabilité climatique

Tableau 5: Valeurs moyennes des paramètres pluviométriques dans les deux sites

\begin{tabular}{|c|c|c|c|c|c|c|c|c|c|c|c|c|c|}
\hline \multicolumn{3}{|c|}{$\begin{array}{l}\text { Fada1 } \\
\text { 1981-1990 }\end{array}$} & \multicolumn{2}{|c|}{ Pama1 } & \multicolumn{2}{|c|}{\begin{tabular}{|l} 
Fada2 \\
$1991-2000$ \\
\end{tabular}} & \multicolumn{2}{|c|}{ Pama2 } & \multicolumn{2}{|c|}{$\begin{array}{l}\text { Fada3 } \\
\text { 2001-2010 }\end{array}$} & \multicolumn{2}{|c|}{ Pama3 } & \multirow[b]{2}{*}{$P^{*}$} \\
\hline & $x$ & $\sigma$ & $x$ & $\sigma$ & $x$ & $\sigma$ & $x$ & $\sigma$ & $X$ & $\sigma$ & $X$ & $\sigma$ & \\
\hline Seqs & 11,0 & 1,8 & 12,2 & 1,6 & 9,3 & 1 & 9 & 1,3 & 9,4 & 1,7 & 10 & 1,6 & $<0,00$ \\
\hline SPI & $-0,9$ & 0,7 & $-0,9$ & 0,9 & 0,1 & 1,2 & 0,3 & 1,2 & $-0,0$ & 0,8 & $-0,1$ & 0,9 & 0,023 \\
\hline $\mathrm{Ht}$ & 718 & 109 & 771 & 137 & 887 & 203 & 965 & 200 & 862 & 131 & 897 & 146 & 0,013 \\
\hline PJrs & 65 & 6 & 46 & 7 & 72 & 5 & 61 & 8 & 69 & 5 & 58 & 5 & $<0,00$ \\
\hline
\end{tabular}

SeqS: séquences sèches (nombre de jours consécutifs sans pluie); SPI: indice standardisé des précipitations; $\mathrm{Ht}$ : pluviométrie (mm); PJrs: nombre de jours pluvieux par an; X: moyenne; $\sigma$ : écart-type.

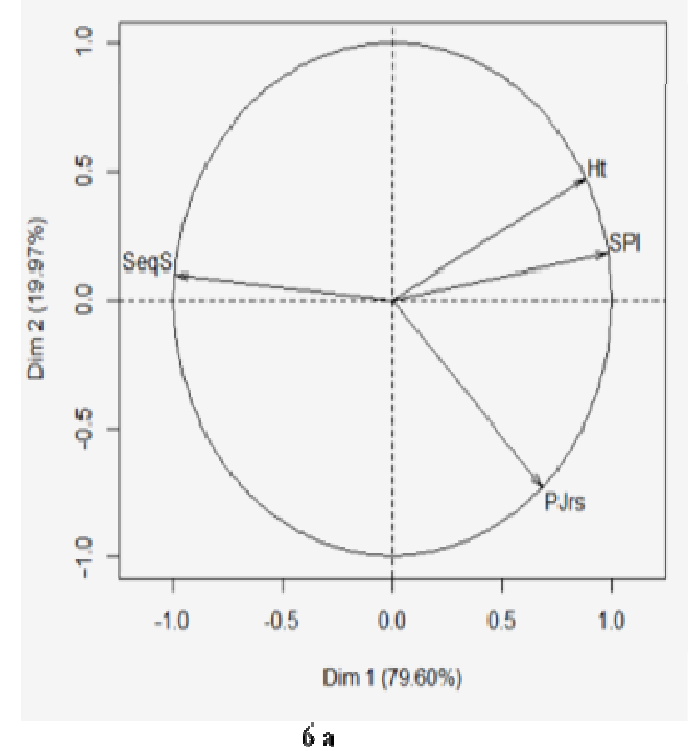

$6 a$

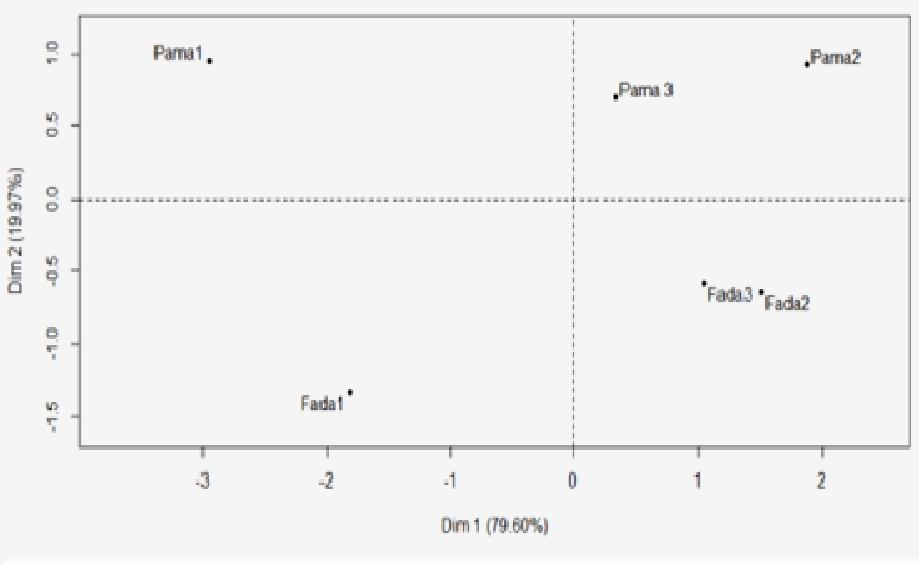

$6 b$

Figures $6 \mathbf{a}$ et $6 \mathbf{b}$ : Cartes factorielles des paramètres pluviométriques $(6 a)$ et des sites selon la période climatiques (6b) Ht: le nombre de jours pluvieux; SPI : Indice standardisé des précipitations ; PJrs: nombre de jours de pluies; SeqS: nombre de jours consécutifs sans pluies;

Fada 1 / Pama 1;Fada 2 / Pama 2; Fada 3 / Pama 3: caractéristiques climatiques de Fada ou de Pama respectivement durant la période 1(1981-1990); période 2 (1991-2000) et période 3 (2001 - 2010)

Types d'utilisation des terres et dégradation des agrosystèmes : L'analyse en composantes principales (ACP) donne un taux de $95,8 \%$ de la variance totale expliquée (Figure 7). La contribution de chaque caractère à la structure des deux principaux axes de l'ACP est donnée dans le Tableau 6. L'axe 1 qui explique $66,7 \%$ de la variance est positivement corrélé avec la densité des ligneux, la densité des individus en régénération, la richesse spécifique moyenne des ligneux, la richesse spécifique moyenne des herbacées et la richesse spécifique moyenne des individus en régénération. Inversement, cet axe 1 est négativement corrélé avec le taux de recouvrement en herbacées, le diamètre moyen des ligneux; la hauteur moyenne des arbustes. De ce fait, l'axe 1 peut être considéré comme exprimant un gradient de dégradation. Ainsi, les forêts communautaires (ZOVIC) contiendraient les unités de végétation les moins dégradées caractérisées par une grande densité des ligneux et des individus en régénération, une grande richesse spécifique moyenne en ligneux, en herbacées et en individus juvéniles. A l'inverse, les jachères (FALL) et les aires de pâturage (GZLD) ont les unités de végétation les plus dégradés, ce qui se reconnaît par un taux d'embuissonnement élevé, un grand diamètre moyen des arbres; un taux élevé de recouvrement en herbacées et une grande 
hauteur des arbustes. La réserve partielle de faune de Pama (FCPN) serait dans un état de dégradation ou de conservation intermédiaire entre les forêts communautaires (ZOVIC) et les deux autres modes d'utilisation des terres. Quant à l'axe 2, il explique 29,1 $\%$ de la variance totale et est corrélé positivement au taux de recouvrement en buissons et négativement à la surface terrière et à la hauteur moyenne des herbacées. Ainsi l'axe 2 expliquerait un gradient de pression anthropique. Les unités d'occupation qui subissent le moins de pression anthropique étant la réserve de Pama, caractérisée par des hautes herbes en saison pluvieuse et une plus grande surface terrière. Les jachères et les aires de pâturage subissent le plus de pression anthropozoïque et cela se traduit par un embuissonnement et aussi une grande richesse spécifique en herbacées.

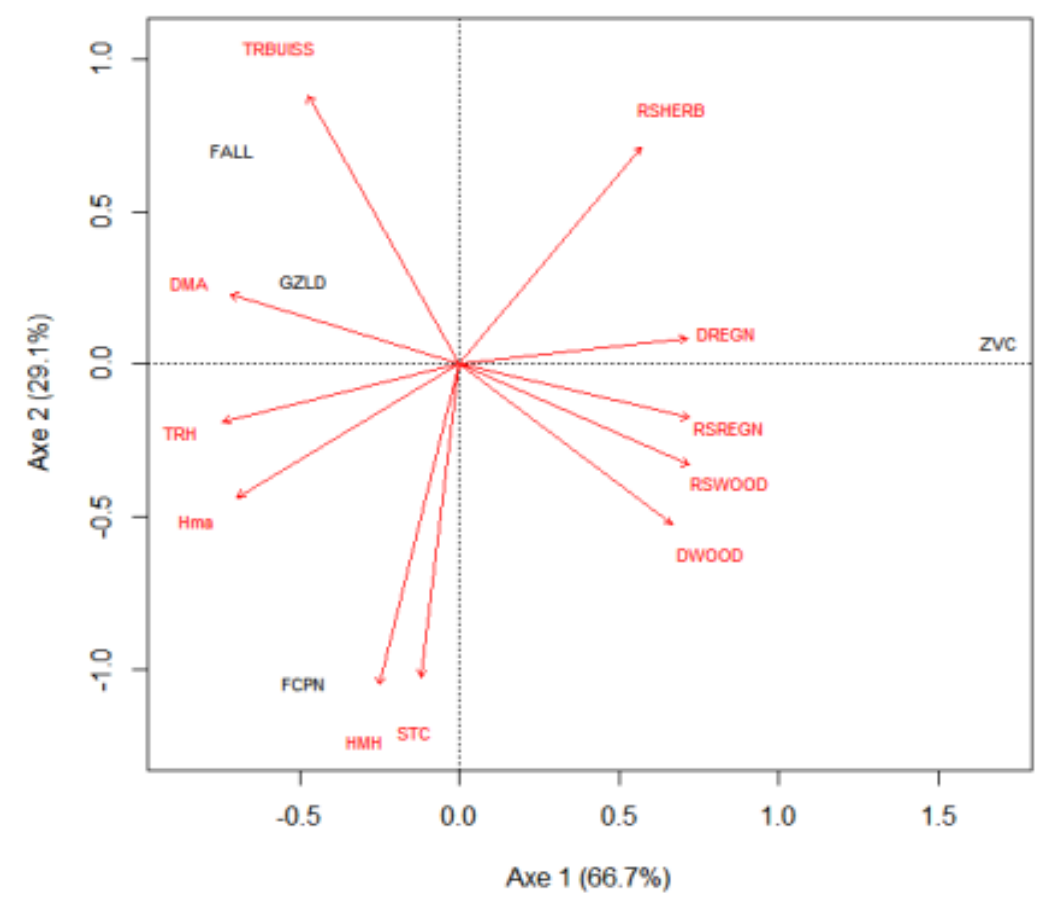

Figure 7 : ACP des sites en fonction des variables écologiques mesurées

FALL: jachère; FCPN: Réserve de Pama; GZLD: aires de pâturage; ZOVIC: zones villageoises d'intérêt cynégétique.

Tableau 6: structure axes de l'ACP

\begin{tabular}{llcc}
\hline Variables & Label & PC1 & PC2 \\
\hline Richesse spécifique moyenne en ligneux & RSWOOD & 0,7 & $-0,2$ \\
Richesse spécifique moyenne en herbacées & RSHERB & 0,6 & 0,5 \\
Richesse spécifique moyenne des régénérations & RSREGN & 0,7 & $-0,1$ \\
Taux de recouvrement des buissons & TRBUISS & $-0,5$ & 0,6 \\
Hauteur moyenne des herbes & HMH & $-0,2$ & $-0,7$ \\
Hauteur moyennes des arbustes & Hma & $-0,7$ & $-0,3$ \\
Taux de couverture herbacée & TRH & $-0,7$ & $-0,1$ \\
Diamètre moyen des arbres & DMA & $-0,7$ & 0,1 \\
Surface terrière & STC & $-0,1$ & $-0,7$ \\
Densité des ligneux & DWOOD & 0,6 & $-0,3$ \\
Densité des individus en régénération & DREGN & 0,7 & 0,1 \\
\hline Proportion cumulative (\%) & & 66,7 & 29,1 \\
\hline
\end{tabular}

\section{DISCUSSION}




\section{Soulama et al. J. Appl. Biosci. Impact des activités anthropiques sur la dynamique de la végétation de la réserve partielle de faune de Pama et de ses périphéries (sud-est du Burkina Faso) dans un contexte de variabilité climatique}

Cartographie : La classification supervisée des images avec huit classes, a donné des valeurs de précision statistiquement acceptables (Kabba \& Li, 2011). Étant donné la complexité du paysage sahélien, un tel résultat s'explique par la qualité des images et le choix des classes thématiques et des zones d'entraînement (Geymen \& Baz, 2008, Maârouhi et al., 2011). En effet, les images sont choisies de telle sorte à éviter la confusion entre les cultures et la végétation naturelle d'une part et d'autre part entre la végétation et les feux de brousse qui réfléchissent dans le proche infra rouge et provoquent des confusion de classes (Koné et al. 2007; Diallo et al., 2011; Mama et al., 2013). L'analyse diachronique des unités d'occupation du sol montre une tendance à la dégradation encore plus exacerbée dans la partie non protégée. Dans la partie anthropisée la dégradation des terres se traduit par une dynamique d'accroissement des sols nus, la réduction de la végétation savanicole et l'augmentation de la classe des mosaïques champs-jachères. Le front agricole a atteint et dégradé la réserve de Pama dans ses limites nord et sud (figure 2). L'analyse de la dynamique spatiale a montré une régression des formations boisées notamment les reliques de forêts claires et les savanes boisées qui sont progressivement remplacées par des savanes arborée ou arbustive. Le même constat est effectué par PRIPODE (2006) dans cette zone. La savane boisée semble être la classe d'occupation la plus affectée par la dégradation car le déboisement affecte $30,8 \%$ du paysage. Cette dynamique régressive est plus accentuée dans les périphéries de la réserve où la conversion des paysages naturels en paysages artificiels accélère le processus de dégradation des terres. La régression de la couverture végétale dans la réserve est davantage due aux facteurs climatiques qu'à l'action de l'homme. La végétation des aires non protégées (jachères, aires de pâturage) est la plus dégradée à cause de l'exacerbation de la pression anthropique. La même tendance est notée dans d'autres pays d'Afrique de l'Ouest, notamment au Mali (Diallo et al., 2011), en Côte d'Ivoire (Kouassi et al., 2012) et au Niger (Mama et al., 2013) où ces auteurs ont montré que les formations denses, particulièrement les savanes boisées, sont les plus affectées par les activités anthropiques et les péjorations climatiques. A l'opposé de cette tendance $66,8 \%$ de la couverture forestière de la zone d'étude connaît une amélioration du couvert végétal dominée par les recrûts forestiers. Cette tendance progressive concerne davantage la réserve de Pama et les ZOVIC que les aires non protégées (jachères et aires de pâturage).

Influence des péjorations climatiques: La période normale allant de 1981 à 2010 connaît la grande sécheresse de 1984 suivie d'une alternance de séquences annuelles humides et sèches avec toutefois une récurrence des séquences sèches. Depuis la fin des années 1970, la récession pluviométrique est observée en Afrique de l'Ouest (Ozer, 2010; Diallo et al., 2011; Kouassi et al., 2012). Cette situation est observée dans notre zone d'étude où on constate une variabilité climatique entre les trois décennies de la normale de référence 1981-2010. Ainsi, les deux dernières décennies de cette normale, bien qu'étant relativement plus humides que la décennie précédente marquée par la grande sécheresse des années 84 , indiquent des variabilités importantes traduites par des séquences sèches de plus en plus récurrentes. La décennie 2001-2010 est relativement plus sèche que la précédente, ce qui pourrait contribuer à expliquer l'état de régression de la phytocénose dans la réserve de Pama et surtout dans les agrosystèmes connexes, constatées dans la Figure 2. La contrainte climatique principale est davantage liée à la variabilité dans la distribution des précipitations et leur imprévisibilité, qu'à la rareté des pluies. C'est la répartition de ces séquences sèches dans le temps qui affecte les écosystèmes car une année de sécheresse, même extrême, peut être moins dramatique qu'une suite de deux ou plusieurs années de sécheresse (Diallo, 2011). Des résultats similaires sont obtenus par d'autres auteurs qui montrent que les effets de la sécheresse en milieu de savane sont observés à travers les variations de la couverture ligneuse qui sont très indicatrices des changements climatiques à l'échelle locale (Koné et al., 2007; Diallo et al., 2011; Kouassi et al., 2012). Contrairement à des études récentes qui tendent à démontrer que la pluviométrie connaît une reprise au Sahel ainsi que la couverture végétale (Hiernaux et al., 2009; Reij et al., 2009), la décennie 2001-2010 (période 3 ) est modérément plus sèche que celle de 1991-2000 (période 2) dans la zone d'étude. Cette sécheresse modérée est encore plus ressentie à Pama (district sud soudanien) qu'à Fada N'Gourma (district nord soudanien). Sachant que la pression anthropique est plus grande dans la zone de Pama (Dipama, 1997; PRIPODE, 2006; Kaboré, 2010), la relative péjoration climatique dans cette zone suggère une interaction entre les facteurs anthropique, climatique et la dégradation des ressources naturelles. 

réserve partielle de faune de Pama et de ses périphéries (sud-est du Burkina Faso) dans un contexte de variabilité climatique

Impacts des types d'utilisation des terres sur la dégradation des ressources naturelles: Selon l'organisation Météorologique Mondiale (OMM, 2005), en plus des facteurs naturels de dégradation des terres, il faut également tenir compte des causes socioéconomiques. II existe une relation entre la dégradation des terres et leur utilisation. Les types d'utilisation des terres se réfèrent au but pour lequel les Hommes destinent les unités d'occupation du sol (Ouédraogo, 2010). L'analyse de la dynamique de l'occupation des sols dans la zone d'étude montre une modification significative de la physionomie des paysages davantage en zone de terroir où la végétation a été considérablement détruite par les défrichements. Ce même constat est effectué par PRIPODE (2006) qui note l'attraction migratoire que ces zones exercent sur les populations des régions défavorisées du pays, la transhumance des animaux et le développement de la culture cotonnière, constituent de sérieux facteurs de dégradation de l'environnement. Les superficies emblavées pour le coton dans la Kompienga sont passées de 1679 ha en 2001 à 4321 ha en 2004 tandis que pour les céréales, ces superficies sont de 14280 à 11169 ha dans le même temps. La pression foncière est plus grande dans la zone de la Kompienga (Pama) que celle du Gourma (Fada N'Gourma). En effet, à cause de la présence du lac du barrage et des terres cultivables, la population de la province de la Kompienga a doublé en une décennie et croît plus vite que celle du Gourma (INSD, 2006; Dipama, 1997). Produits de la progression du front agro-pastoral, les hameaux de culture sont aussi importants que des villages administratifs reconnus et le moindre relâchement du contrôle forestier se traduit presque systématiquement par l'installation de champs et ou de villages dans ces zones. Les populations s'adonnent à des méthodes culturales non autorisées telles que l'exploitation agricole des sols fragiles (érodibles ou pentus), les cultures sur brûlis, les feux de brousse, la coupe du bois et le pâturage clandestin (Kaboré, 2010). Ainsi, l'espace agro-pastoral officiel est confiné entre la réserve de Pama; le lac de la Kompienga et les ZOVIC. On estime à $80 \%$ environ la part absorbée actuellement par la réserve sur l'ensemble de la superficie de la province de la Kompienga. Nos investigations sur le terrain ont montré que les jachères deviennent encore plus rares dans la province de la Kompienga que dans celle du Gourma. Cette situation explique la diminution de la classe des mosaïques champs-jachères tandis que celle des sols nus et affleurement rocheux augmente (Roose, 1991). La diminution de la classe mosaïques champs-jachères dans la réserve de partielle de faune de Pama suivie de son boisement, s'explique par les déguerpissements des populations situées à l'intérieur de la réserve. En effet Kaboré (2010) mentionne le déguerpissement d'une quarantaine de localités des bords de la rivière dite Bouboanga en 1984 et le départ de plus de 1000 habitants d'une dizaine de villages et hameaux de culture en 1993. La grande pression foncière a conduit à mettre en œuvre la notion de zones tampons traduite par la création des ZOVIC afin de protéger la réserve de Pama. L'action a consisté à " négocier » avec les populations limitrophes la désignation des terroirs bordant le parc comme « zones villageoises d'intérêt cynégétiques » et de définir des règles d'usage des ressources allant dans le sens de la « préservation de la biodiversité » (Kaboré, 2010). En plus de l'agriculture, l'élevage apparaît comme une activité accentuant la dégradation des terres dans cette région. La zone d'étude est caractérisée par le surpâturage aggravée par la transhumance interne et internationale (Kpoda, 2010; Sawadogo et al., 2014). Au Burkina Faso, la culture fourragère demeure marginale et la base essentielle de l'alimentation des animaux reste le fourrage naturel, auquel s'ajoutent les résidus des récoltes. La capacité de charge des prairies est dépassée vue leur physionomie caractéristiques (sols nus, compactés, embuissonnement.). Le surpâturage entraîne la réduction de la régénération naturelle des ligneux, la baisse de la couverture herbacée, la mise à nue du sol et son endurcissement (Kessler \& Geerling, 2006). A l'inverse, le rôle du pâturage sur l'enrichissement spécifique des espaces ouverts a été démontré d'où la valeur de la richesse spécifique moyenne en herbacée plus élevée dans les endroits pâturés (aires de pâturage, jachères, ZOVIC). Les herbacées sont indicatives des changements environnementaux d'un site à court terme (Devineau, 1999; Bangirinama, 2010). Le processus de développement de la savane boisée dans la réserve peut également s'expliquer par l'impact des feux précoces (Louppe et al., 1998). La zone non protégée est soumise à la pression du pâturage et des cultures si bien que la forêt et la savane dense n'ont pas le temps de se développer. Cela traduit une difficulté d'adaptation du système agraire dominant dans cette zone (Hiernaux, 1998; Devineau et al., 2010). En effet, l'intensification de l'agriculture implique la maîtrise de certaines innovations technologiques (variétés 
améliorées, mécanisation, techniques d'aménagement des sols et des eaux, etc.). Malgré la préservation des arbres utiles dans les champs, la destruction totale des autres arbres ne favorise pas la reconstitution de la végétation originelle. Seuls les arbustes sont capables de se régénérer rapidement. II s'en suit alors une modification de la structure et de la composition de la végétation avec l'installation d'espèces pionnières ou rudérales. La surexploitation des terres est considérée comme la principale cause de la dégradation du couvert végétal et de la perte en biodiversité (Diallo et al., 2011; Mama et al., 2013).

Conclusion et implication pour la conservation : La dégradation des terres au cours de la période 20012013, dans la réserve partielle de faune de Pama et les agrosystèmes connexes, apparaît comme une conséquence des effets combinés de l'Homme et des péjorations climatiques, notamment la sécheresse. La réduction de la végétation et l'ouverture du paysage apparaissent comme étant les deux grandes formes de dégradation des terres dans cette zone. La quantification du changement du paysage grâce à la télédétection et au Système d'Information Géographique, montre une amélioration $(58,25 \%)$ i.e. un léger accroissement du couvert forestier ou $d u$ matériel sur pied dans la réserve partielle de faune de

\section{REMERCIEMENTS}

Cette étude a été conduite grâce au projet UNDESERT (Understanding and Combating desertification to mitigate its impact on Ecosystem services), financé par I'Union Européenne (EU FP7 243906). Nous exprimons

\section{RÉFÉRENCES}

Ariori SL, Ozer P, 2005. Évolution des ressources forestières en Afrique de l'Ouest soudanosahélienne au cours des 50 dernières années. Geo-Eco-Trop, 29: 61-68.

Aubreville A, 1957. Accord de Yangambi sur la nomenclature des types africains de vegetation. Bois et Forêts des Tropiques, 51 : 23-7.

Bangirinama F, Bigendako MJ, Lejoly J, Noret N, De Nannière C, Bogaert J, 2010. Les indicateurs de la dynamique post-culturale de la végétation des jachères dans la partie savane de la réserve naturelle forestière de Kigwena (Burundi). Plant Ecology and Evolution , 143: 138-147.
Pama tandis que dans la partie non protégée, la dégradation domine. Elle est de $28,82 \%$ pour toute la zone. L'anthropisation perturbe l'équilibre écologique de la végétation de cette région. La matrice de transition a montré le déclin des formations denses en particulier la savane boisée qui est la classe d'occupation la plus affectée par la dégradation tandis que la savane arbustive et arborée se développe. A côté des modes d'utilisation des ressources (agriculture et élevage) qui n'obéissent pas à des normes de durabilité, le climat est aussi un facteur de perturbation du milieu. En effet, nos résultats corroborent l'idée de l'aridification du climat dans les régions sèches à travers des variabilités importantes traduites par des séquences sèches de plus en plus récurrentes. II est bien vrai que dans cette étude des insuffisances demeurent pour démêler les effets conjoncturels de la sécheresse de l'impact humain. De plus les impacts humains sont quelque peu obscurcis par la variabilité (topographie; types de sol; types de végétation). Les aires protégées de la zone d'étude contribuent efficacement à protéger les ressources végétales bien qu'il soit nécessaire de redoubler la surveillance face à la pression foncière. Par ailleurs il faut sensibiliser et former les populations rurales à l'utilisation de techniques culturales durables.

notre gratitude aux guides de terrain Niada; Koidima; Yombo; aux agents des eaux et forêts Soubeiga Bertrand.

Bergaoui M, Alouini, A, 2001. Caractérisation de la sécheresse météorologique et hydrologique: cas du bassin versant de Siliana en Tunisie. Sécheresse, 12: 205-13.

Devineau JL, 1999. Rôle du bétail dans le cycle culturejachère en région soudanienne : la dissémination d'espèces végétales colonisatrices d'espaces ouverts (Bondoukuy, Sud-ouest du Burkina Faso). Rev. Ecol., 54: 97-121.

Devineau, JL, Fournier A, Nignan S, 2010. Savanna fire regimes assessment with MODIS fire data : their relationship to land cover and plant species distribution in western Burkina Faso (West Africa). J. Arid Envir., 74 : 1092-1101. 
Diallo H, Bamba I, Barima YSS, Visser M, Ballo A, Mama A, Vranken I, Maïga $M$, Bogaert J, 2011. Effets combines du climat et des pressions anthropiques sur la dynamique évolutive de la vegetation d'une zone protégée du Mali (Réserve de Fina, Boucle du Baoulé).Sècheresse, 22:97-107.

Dipama JM, 1997. Les impacts du barrage hydroélectrique sur le bassin versant de la Kompienga (Burkina Faso). Thèse de doctorat de l'Université de Bordeaux III, Option Géographie tropicale. P. 392.

FAO, 2008. La dégradation des sols s'intensifie. www.FAO. Org 4 Août 2010.

FAO 2001. Définitions de bases relatives à l'évaluation des ressources forestières en 2000. http://www.fao.org/docrep/meeting/003/X9835f /X9835f01.htm.

Fontès J, Guinko S. 1995. Carte de la végétation et de l'occupation du sol du Burkina Faso. Centre national de la recherche scientifique de l'université de Toulouse III, Institut de développement rural, Faculté des sciences et techniques de l'université de Ouagadougou, Ministère de la coopération Française, Projet campus $88313101.65 p$.

Geymen A, Baz I, 2008. The potential of remote sensing for monitoring land cover changes and effects on physical geography in the area of Kayisdagi mountain and its surroundings (Istanbul). Environmental Monitoring and Assessment, 140 (1-3) : 33-42.

Hahn-Hadjali K, 1998. Les groupements végétaux des savanes du sud-est du Burkina Faso (Afrique de l'Ouest). Études sur la flore et la végétation du Burkina Faso et des pays avoisinants, 3: 379.

Hiernaux P, Diarra L, Trichon V, Mougin E, Soumaguel N, Baup F, 2009. Woody plant population dynamics in response to climate changes from 1984 to 2006 in Sahel (Gourma, Mali). Journal of Hydrology, 375 (1-2), 103-113.

Hiernaux $P, 1998$. Effects of grazing on plant species composition and spatial distribution in rangelands of the Sahel. Plant Ecol., 138 : 191-202.

http://populationenvironmentresearch.org/pape rs/BurkinaFaso_Nov2006.pdf, consulté le 03 octobre 2014.
INSD, 2006. Institut National des Statistiques et de la Démographie. Recensement général de la population et de l'Habitation (RGPH) 1985, 1996, 2006.

Kabba STV, Li J, 2011. Analysis of land use and land cover changes, and their ecological implication in Wuhan, China. Journal of Geography and Geology, 3: 104-118.

Kaboré $A, 2010$. Brousse des uns, aire protégée des autres, histoire du peuplement, perceptions de la nature et politique des aires protégées dans le Gourma burkinabè: l'exemple de la Réserve partielle de faune de Pama. Thèse de doctorat présentée à l'Institut de Hautes Études Internationales et du Développement, Genève, Suisse, 383p + annexes.

Kessler J, Geerling C, 2006. Profil Environnemental du Burkina Faso, rapport final. Université agronomique de Wageningen, Pays-Bas. http://ec.europa.eu/development/icenter/reposi tory/burkina_faso_CEP_2006.pdf

Koné M, Aman A, Adou-Yao CY, Coulibaly L, N'guessan K E, 2007. Suivi diachronique par télédétection spatiale de la couverture ligneuse en milieu de savane soudanienne en Côte d'Ivoire. Revue Télédétection, 7: 433-446.

Kouassi AM ; Kouamé KF, Ahoussi KE, Oularé S, Biemi $\mathrm{J}$, 2012. Impacts conjugués des changements climatiques et des pressions anthropiques sur les modifications de la couverture végétale dans le bassin versant du N'zi-Bandama (Côte d'Ivoire). Rev. Ivoir. Sci. Technol., 20: 124 146

Kpoda C, 2010. Effet du changement climatique sur les modes de conduite du bétail. Vulnérabilité et adaptation des éleveurs du Burkina Faso. Mémoire d'ingénieur, option élevage, IDR/UPB, 93p.

Larwanou M, Saadou M, Nonguierma A, 2005. Détermination du degré d'aridité bioclimatique de sept localités du département de Tillabéri (sud-ouest du Niger) : classement en zones bioclimatiques. Science et changements planétaires / Sécheresse, 16 (2): 107-114.

Louppe D, Olivier R, Ouattara N, Fortier M, 1998. Impacts des feux répétés sur les sols de la savane du centre de la Côte d'Ivoire. Communication au Séminaire International de Ouagadougou sur l'Aménagement intégré des forêts naturelles 
des zones tropicales sèches de l'Afrique de l'Ouest: 161-173.

Maârouhi IM, Mahamane A, Saadou M, Bachmann Y, 2011. Dynamique spatio-temporelle des forêts claires dans le Parc national du W du Niger (Afrique de l'Ouest). Sècheresse, 22: 97-107.

Mama A, Sinsin B, De Cannière C, Bogaert J, 2013. Anthropisation et dynamisation des paysages en zone soudanienne au nord du Bénin. TROPICULTURA, 31 (1): 78-88.

Mbaygone E, T hiombiano A, Hahn-Hadjali K, Guinko $S, 2008$. Structure des ligneux des formations végétales de la Réserve de Pama (Sud-Est du Burkina Faso, Afrique de l'Ouest). Flora et Vegetatio Sudano- Sambesica, 11: 25-34.

Nacoulma BMI, Traoré S, Hahn K, Thiombiano A, 2011. Impact of land use types on population structure and extent of bark and foliage harvest of Afzelia africana and Pterocarpus erinaceus in Eastern Burkina Faso. International Journal of Biodiversity and Conservation, 3(3): 62-72.

Nicholson S, 2005. On the question of the "recovery" of the rains in the West African Sahel. Journal of Arid Environments, 63: 615-641.

Olsson L, Eklundhb L, Ardo J, 2005. A recent greening of the Sahel-trends, patterns and potential causes. Journal of Arid Environments, 63 : 556-566.

OMM, 2005, Organisation Mondiale de la Météorologie. Le climat et la dégradation des sols. TempsClimat-Eau. OMM-n0989. 34 p.

Ouédraogo I, Savadogo $\mathrm{P}$, Tigabu M, Cole R, Odén PC, Ouadba JM, 2009. Is rural migration a threat to environmental sustainability in southern Burkina Faso? Land Degradation \& Developement, 20(2): 217-230.

Ouédraogo I, 2010. Land use Dynamics and Demographic Change in Southern Burkina Faso. Doctoral thesis N0 2010:63, Faculty of Forest Sciences, southern Swedish Forest Research Centre Alnap. 64 P. + annexes.

Ouédraogo O, Thiombiano A, Hahn-Hadjali K, Guinko $S$, 2008. Diversité et structure des groupements ligneux du parc national de Arly (Est du Burkina Faso). Flora et Vegetatio Sudano-Sambesica, 11:5-16.

Ouédraogo $\mathrm{O}$, Thiombiano A, Hahn-Hadjali K, Guinko $S, 2009$. Diversité et dynamique de la végétation ligneuse juvénile du Parc National d'Arly (Burkina Faso). Candollea, 64: 257-278.

Ozer P, Hountondji YC, Niang AJ, Karimoune S, Manzo OL, Salmon M, 2010. Désertification au Sahel: historique et perspectives. BSGLg , 54:69- 84

Paré S, Söderberg U, Sandewall M, Ouadba JM, 2010. Land use analysis from spatial and field data capture in southern Burkina Faso, West Africa. Agriculture, Ecosystems \& Environment, 127: 277-285.

Pontius, RG, 2000. Quantification error versus location in comparison of categorical maps. Photogrammetric Engineering and Remote Sensing, vol. 66, (8): 1011-1016.

PRIPODE, 2006. Programme International de Recherche sur les Interactions entre la Population, le Développement et l'Environnement. Mobilité spatiale de la population: nécessité de développement et risques de dégradation de l'environnement dans l'Est et le Sud-Ouest du Burkina Faso. P.57.

Reij C, Tappan G, Smale M, 2009. Re-greening the Sahel: farmer-led innovation in Burkina Faso and Niger. Millions fed: proven successes in agricultural development, 53-58.

Reynolds, J.F., Stafford Smith, M, 2002. Global desertification: do humans create deserts? In: Stanfford-Smith, M., Reynolds, J.F. (Eds.), Do Humans Create Deserts? Dahlem University Press, Berlin, pp. 1-22.

Roose, E., 1991. Capacité des jachères à restaurer la fertilité des sols pauvres en zone soudanosahélienne d'Afrique Occidentale. In : la jachère en Afrique de l'Ouest. Atelier international, Montpellier du 2 au 5 décembre, O.R.S.T.O.M, Paris, pp 233-243.

Sawadogo I, Devineau JL, Fournier A, 2014. État des ressources pastorales dans une terre d'accueil et de transit des pasteurs transhumants: le terroir de Kotchari (sud-est du Burkina Faso).

Schmid S, 2003. Les images satellites multitemporelles comme outil d'analyse du couvert végétal: le cas des savanes du sud du Burkina Faso (Afrique Occidentale). Études sur la flore et la végétation du Burkina Faso et des pays avoisinants, 7: 31-36.

Shalaby A, Tateishi R, 2007. Remote sensing and GIS for mapping and monitoring land cover and land-use changes in the Northwestern coastal 

réserve partielle de faune de Pama et de ses périphéries (sud-est du Burkina Faso) dans un contexte de variabilité climatique

zone of Egypt. Applied Geography, 27 (1): 2841.

Sinsin B, 1993. Phytosociologie, écologie, valeur pastorale, production et capacité de charge des pâturages naturels du périmètre NikkiKalalé au Nord-Bénin. Thèse présentée en vue de l'obtention du grade de Docteur en Sciences Agronomiques. Université Libre de Bruxel.

Tabopda GW, Fotsing JM, 2010. Quantification de l'évolution du couvert végétal dans la réserve forestière de Laf-Madjam au nord du Cameroun par télédétection satellitale. Sécheresse, 21: 169-78.

UICN, 2009. (Union Internationale pour la Conservation de la Nature). Parcs et réserves du Burkina Faso, Évaluation de l'efficacité de la gestion des aires protégées. 81p.

Youl S, Barbier B, Moulin CH, Manlay RJ, Botoni E, Masse D, Hien V, Feller C, 2008. Modélisation empirique des principaux déterminants socioéconomiques de la gestion des exploitations agricoles au sud-ouest du Burkina Faso. Biotechnologie Agronomie Société et Environnement, 12(1): 9-21. 